\title{
Remarks on the existence of Cartier divisors
}

\author{
Stefan Schröer
}

March 16, 2005

\begin{abstract}
We characterize those invertible sheaves on a noetherian scheme which are definable by Cartier divisors and correct an erroneous counterexample in the literature.
\end{abstract}

\section{Introduction}

Let $X$ be a noetherian scheme and $\mathcal{L}$ an invertible $\mathcal{O}_{X}$-module; does there exist a Cartier divisor $D \in \operatorname{Div}(X)$ with $\mathcal{L} \simeq \mathcal{O}_{X}(D)$ ? This is no problem if $X$ satisfies Serre's condition $\left(S_{1}\right)$, and the issue is to deal with embedded components. The goal of this short note is to provide an answer and to correct an erroneous counterexample in the literature.

The question was first posed by Nakai [5, p. 300], and later Grothendieck $[3,21.3 .4]$ showed that the canonical map $\operatorname{Div}(X) \rightarrow \operatorname{Pic}(X)$ is surjective if the subset $\operatorname{Ass}\left(\mathcal{O}_{X}\right) \subset X$ allows an affine open neighborhood. On the other hand, it seemed to be well known from the beginning that in general obstructions might arise. Hartshorne proposed a construction (attributed to Kleiman) of a non-projective irreducible 3 -fold $X$ with a single embedded component $x \in X$ for which it is claimed that $\operatorname{Div}(X) \rightarrow \operatorname{Pic}(X)$ is not surjective $\left[4\right.$, ex. 1.3, p. 9]. Unfortunately, $\operatorname{Ass}\left(\mathcal{O}_{X}\right)=\{x, \eta\}$ is contained in every affine open neighborhood $U \subset X$ of $x$, and Grothendieck's criterion tells us that that the proposed construction does not yield an invertible sheaf without Cartier divisor.

Key words: Cartier divisor, invertible sheaf. Mathematics subject classification (1991): 14C20 
In the first part of this note we will discuss how the construction can be modified in order to obtain the desired counterexample. In the second part we will prove a positive result, which complements Grothendieck's criterion in the following way: Let $T \subset X$ be a finite subset containing $\operatorname{Ass}\left(\mathcal{O}_{X}\right)$; then there is a Cartier divisor $D \in \operatorname{Div}(X)$ with $\mathcal{L} \simeq \mathcal{O}_{X}(D)$ and support $\operatorname{Supp}(D)$ disjoint from $T$ if and only if the restriction of $\mathcal{L}$ to $T$ is trivial. Here we view $T$ also as a ringed space, endowed with the subspace topology and sheaf of rings $\mathcal{O}_{T}=i^{-1}\left(\mathcal{O}_{X}\right)$, where $i: T \rightarrow X$ is the inclusion map.

\section{Absence of Cartier divisors}

In this section we construct two schemes $X$ for which $\operatorname{Div}(X) \rightarrow \operatorname{Pic}(X)$ is not surjective.

(2.1) Let us recall Hartshornes construction. We fix a ground field $K$; then there is a regular, integral, proper 3-fold $Y$ containing two irreducible curves $A, B \subset Y$ such that $A+B$ is numerically trivial. Such a scheme is obviously non-projective, and was constructed by Hironaka using local blow-ups; the construction is thoroughly discussed in [6, p. 75]. For each Cartier divisor $D \in \operatorname{Div}(Y)$ we have

$$
A \cdot D>0 \Leftrightarrow B \cdot D<0
$$

and the complement of an affine open neighborhood $U \subset Y$ of the generic point of $A$ defines such a Cartier divisor. Choose a closed point $a \in A$ and consider the infinitesimal extension $Y \subset X$ with ideal $\mathcal{I}=\kappa(a)$. The outer groups in the exact sequence

$$
H^{1}(Y, \mathcal{I}) \longrightarrow \operatorname{Pic}(X) \longrightarrow \operatorname{Pic}(Y) \longrightarrow H^{2}(Y, \mathcal{I})
$$

vanishes, hence there is an invertible $\mathcal{O}_{X^{-}}$-module $\mathcal{L}$ with $B \cdot c_{1}(\mathcal{L})>0$. Grothendieck's criterion tells us that $\mathcal{L}$ is representable by a Cartier divisor $D \in \operatorname{Div}(X)$; assume that it is even representable by an effective Cartier divisor $D \subset X$. But $A \cdot D<0$ implies $A \subset D$, hence $a \in D$; on the other hand, according to $[2,3.1 .9], D$ must be disjoint to $\operatorname{Ass}\left(\mathcal{O}_{X}\right)$, contradiction. In other words, the construction only yields a Cartier divisor $D \in \operatorname{Div}(X)$ not linearly equivalent to an effective one such that the restriction to $X^{\text {red }}=Y$ is equivalent to an effective Cartier divisor.

In order to achieve the desired effect we have to introduce at least two embedded components. Choose closed points $a \in A$ and $b \in B$, and let 
$Y \subset X$ be the infinitesimal extension with ideal $\mathcal{I}=\kappa(a) \oplus \kappa(b)$. Again there is an invertible $\mathcal{O}_{X}$-module $\mathcal{L}$ with $A \cdot c_{1}(\mathcal{L})<0$ and $B \cdot c_{1}(\mathcal{L})>0$. We observe that $\operatorname{Div}(X) \subset Z^{1}(X)$ is the subgroup generated by all prime cycles disjoint to $\{a, b\}$. Assume that there is a Cartier divisor $D \in \operatorname{Div}(X)$ representing $\mathcal{L}$. Decomposing $D=\sum n_{i} D_{i}$ into prime cycles, we see that each summand is Cartier, hence $A \cdot D_{i} \neq 0$ and $B \cdot D_{i} \neq 0$ holds for some index $i$. Consequently we have $A \cdot D_{i}<0$ and $a \in D_{i}$, or $B \cdot D_{i}<0$ and $b \in D_{i}$; in both cases, $\operatorname{Ass}\left(\mathcal{O}_{X}\right)=\{a, b, \eta\}$ is not disjoint to $D_{i} \subset X$, contradiction. Hence it is impossible to represent $\mathcal{L}$ by a Cartier divisor.

(2.2) Another counterexample features non-separated schemes. Let $A$ be a discrete valuation ring with field of fraction $R$. We can glue two copies $U_{1}, U_{2}$ of $\operatorname{Spec}(A)$ along $\operatorname{Spec}(R)$ and obtain an integral, regular curve $Y$, which is a non-separated scheme $[1,8.8 .5]$. The group $\operatorname{Div}(Y)=Z^{1}(Y)$ is isomorphic to $\mathbb{Z}^{2}$, and the exact sequence

$$
1 \longrightarrow \Gamma\left(Y, \mathcal{O}_{Y}\right)^{\times} \longrightarrow \Gamma\left(Y, \mathcal{M}_{Y}\right)^{\times} \longrightarrow \operatorname{Div}(Y) \longrightarrow \operatorname{Pic}(Y) \longrightarrow 0
$$

yields $\operatorname{Pic}(Y)=\mathbb{Z}$. Let $Y \subset X$ be the infinitesimal extension with the ideal $\mathcal{I}=\kappa\left(y_{1}\right) \oplus \kappa\left(y_{2}\right)$, where $y_{1}, y_{2} \in Y$ are the closed points. The restriction map $\operatorname{Pic}(X) \rightarrow \operatorname{Pic}(Y)$ is bijective, but the sheaf $\operatorname{Div}_{X}$ is zero. Thus we have $\operatorname{Div}(X)=0$, and $\mathcal{L}=\mathcal{O}_{X}$ is the only invertible sheaf associated to a Cartier divisor.

\section{Existence of Cartier divisors}

In this section, $X$ is a noetherian scheme, and $T \subset X$ is a finite subset containing the finite subset $\operatorname{Ass}\left(\mathcal{O}_{X}\right) \subset X$.

(3.1) Let $\operatorname{Div}_{T}(X) \subset \operatorname{Div}(X)$ be the subgroup of Cartier divisors $D$ with $\operatorname{Supp}(D) \cap T=\emptyset$. Recall that the support $\operatorname{Supp}(D)$ is defined as the support of $D_{1} \cup D_{2}$, where cyc $(D)=D_{1}-D_{2}$ is the decomposition into positive and negative parts of the associated Weil divisor.

This construction can be sheafified: Let $\mathcal{S}_{T} \subset \mathcal{O}_{X}$ be the subsheaf of sets whose stalk $\mathcal{S}_{T, x}$ consists of the stalks $s_{x} \in \mathcal{O}_{X, x}$ whose localizations $s_{y} \in \mathcal{O}_{X, y}$ are units for all $y \in \operatorname{Spec}\left(\mathcal{O}_{X, x}\right) \cap T$. Let $\mathcal{M}_{X, T}=\mathcal{S}_{T}^{-1} \mathcal{O}_{X}$ be the localization in the category of sheaves of rings. We now define a sheaf of abelian groups $\mathcal{D} i v_{X, T}$, written additively, by the exact sequence

$$
1 \longrightarrow \mathcal{O}_{X}^{\times} \longrightarrow \mathcal{M}_{X, T}^{\times} \longrightarrow \mathcal{D i v}_{X, T} \longrightarrow 0
$$


and obtain $\operatorname{Div}_{T}(X)=\Gamma\left(X, \operatorname{Div}_{X, T}\right)$. Now let $i: T \rightarrow X$ be the inclusion map, and set $\mathcal{O}_{T}=i^{-1}\left(\mathcal{O}_{X}\right)$. We observe the following

(3.2) Proposition. The $\mathcal{O}_{X}$-algebras $\mathcal{M}_{X, T}$ and $i_{*}\left(\mathcal{O}_{T}\right)$ are canonically isomorphic.

First, assume that $X$ is the spectrum of a local ring $A$ with closed point $x \in X$. Let $S \subset A$ be the multiplicative subset of all $a \in A$ with $a / 1 \in A_{\mathfrak{p}}^{\times}$ for all primes $\mathfrak{p} \subset A$ corresponding to points $t \in T$. Clearly, $i_{*}\left(\mathcal{O}_{T}\right)_{x}$ and $\left(\mathcal{M}_{X, T}\right)_{x}$ are canonically isomorphic to $S^{-1} A$. In the general case, consider the diagram

$$
\begin{gathered}
i_{*}\left(\mathcal{O}_{T}\right) \longrightarrow \prod_{x \in X} i_{*}\left(\mathcal{O}_{T}\right)_{x} \\
\downarrow \\
\mathcal{M}_{X, T} \longrightarrow \\
\prod_{x \in X}\left(\mathcal{M}_{X, T}\right)_{x},
\end{gathered}
$$

where the horizontal maps are the canonical inclusions. Since the bijections $i_{*}\left(\mathcal{O}_{T}\right)_{x} \rightarrow\left(\mathcal{M}_{X, T}\right)_{x}$ are compatible with localization, the vertical map induces the desired bijection $i_{*}\left(\mathcal{O}_{T}\right) \rightarrow \mathcal{M}_{X, T}$. QED.

It should be noted that these $\mathcal{O}_{X}$-algebras are in general not quasicoherent. From the above fact we immediately obtain the following criterion:

(3.3) Theorem. An invertible $\mathcal{O}_{X}$-module $\mathcal{L}$ is representable by a Cartier divisor $D \in \operatorname{Div}(X)$ with support disjoint from $T$ if and only if the restriction of $\mathcal{L}$ to $T$ is trivial in $\operatorname{Pic}(T)$.

Let $i: T \rightarrow X$ be the corresponding flat morphism of ringed spaces. The exact sequence (3.1.1) can be rewritten as

$$
1 \longrightarrow \mathcal{O}_{X}^{\times} \longrightarrow i_{*} i^{*}\left(\mathcal{O}_{X}^{\times}\right) \longrightarrow \mathcal{D i v}_{X, T} \longrightarrow 0,
$$

and we obtain an exact sequence

$$
\operatorname{Div}_{T}(X) \longrightarrow \operatorname{Pic}(X) \longrightarrow H^{1}\left(X, i_{*} i^{*}\left(\mathcal{O}_{X}^{\times}\right)\right) .
$$

The spectral sequence for the composition $\Gamma \circ i_{*}$ gives an inclusion

$$
0 \longrightarrow H^{1}\left(X, i_{*}\left(\mathcal{O}_{T}^{\times}\right)\right) \longrightarrow H^{1}\left(T, \mathcal{O}_{T}^{\times}\right),
$$

and we end up with the exact sequence

$$
\operatorname{Div}_{T}(X) \longrightarrow \operatorname{Pic}(X) \longrightarrow \operatorname{Pic}(T),
$$

which is precisely our assertion. QED. 
(3.4) Remark. Grothendieck's criterion can be recovered from this: Assume that $T \subset X$ is contained in an affine open neighborhood $U=\operatorname{Spec}(A)$. If $S \subset A$ is the complement of the union of all primes $\mathfrak{p} \subset A$ corresponding to points $x \in U \cap T$, then $T$ is also contained in the semi-local scheme $V=\operatorname{Spec}\left(S^{-1} A\right)$, and $\operatorname{Pic}(X) \rightarrow \operatorname{Pic}(T)$ factorizes over $\operatorname{Pic}(V)$. Since the Picard group of a semi-local ring vanishes, each invertible $\mathcal{O}_{X}$-module is representable by a Cartier divisor $D \in \operatorname{Div}_{T}(X)$.

\section{References}

[1] A. Grothendieck, J.A. Dieudonné: Eléments de géométrie algébrique I: Le language de schémas. Springer-Verlag, Berlin etc. 1970 .

[2] A. Grothendieck: Eléments de géométrie algébrique IV: Etuede locale des schémas et de morphismes de schémas. Publ. Math. IHES 24 (1965).

[3] A. Grothendieck: Eléments de géométrie algébrique IV: Etuede locale des schémas et de morphismes de schémas. Publ. Math. IHES 32 (1967).

[4] R. Hartshorne: Ample vector bundles. Publ. Math. IHES 29 (1966), 63-94

[5] Y. NAKAI: Some fundamental lemmas on projective schemes. Trans. Am. Math. Soc. 109 (1963), 296-302.

[6] I. Shafarevich: Basic algebraic geometry. 2: Schemes and complex manifolds. Springer, Berlin etc., 1994.

Anschrift des Autors:

Mathematisches Institut

Ruhr-Universität

44780 Bochum

Germany

E-mail s.schroeer@ruhr-uni-bochum.de 